\title{
EL PERFIL DEL TUTOR DE TITULACIÓN DE TERCER NIVEL. CASO: FACULTAD DE CIENCIAS ADMINISTRATIVAS DE LA UNIVERSIDAD DE GUAYAQUIL, ECUADOR
}

\section{THE PROFILE OF THE THIRD LEVEL DEGREE TUTOR. CASE: FACULTY OF ADMINISTRATIVE SCIENCES OF UNIVERSIDAD DE GUAYAQUIL, ECUADOR}

José Alonso Hidalgo León, MSc.

Máster universitario en Planificación y Gestión de Destinos y Productos Turísticos (Ecuador).

Docente Titular Agregado en la Carrera de Turismo de la Facultad de Ciencias

Administrativas de la Universidad de Guayaquil, Ecuador.

orcid.org/0000-0002-5499-1351

jose.hidalgo@uq.edu.ec

Edison Fernando Chiriboga Cisneros, MSc.

Máster en Administración de Empresas (Ecuador).

Docente Titular Agregado en la Carrera de Turismo de la Facultad de Ciencias

Administrativas de la Universidad de Guayaquil, Ecuador.

orcid.org/0000-0002-5764-8065

edison.chiribogac@uq.edu.ec

\section{ARTÍCULO DE INVESTIGACIÓN}

Recibido: 2 de septiembre de 2019.

Aceptado: 18 de noviembre de 2019.

\section{RESUMEN}

La presente investigación tiene por objetivo determinar el perfil del tutor de titulación de tercer nivel tomando el caso de la Facultad de Ciencias Administrativas de la Universidad de Guayaquil en Ecuador. Consecuentemente se aplicó la técnica de grupo focal con los gestores del área de titulación de las 7 carreras vigentes para otorgamiento de títulos, además se realizó una encuesta a 115 docentes tutores de titulación de la facultad que descargan horas en el área respectiva. El perfil del docente-tutor se compone de 3 ejes principales: conocimiento, competencia y experiencia. 
Palabras clave: docente-tutor, gestión universitaria, criterios, proceso de titulación.

\section{ABSTRACT}

The purpose of this research is to determine the profile of the third level advisor taking the case of the Faculty of Administrative Sciences of the University of Guayaquil in Ecuador. Consequently, the focus group technique was applied with the managers of the respective area of the 7 current available careers, in addition a survey was conducted of 115 faculty advisors. The advisor profile consists of 3 main axes: knowledge, competence and experience.

Keywords: teacher tutor, university management, criteria, degree process.

\section{INTRODUCCIÓN}

En la última década, se han evidenciado cambios relevantes y significativos en la educación superior ecuatoriana. Los cuatro periodos presidenciales del partido Alianza País durante la denominada Revolución Ciudadana han enrumbado a las entidades universitarias ecuatorianas en el camino a la excelencia, considerando áreas sustanciales de la gestión universitaria como son la docencia, vinculación con la colectividad, investigación e innovación, entre otras. De esta premisa gubernamental parte el proyecto de investigación $\mathrm{FCl}$ denominado "La gestión del conocimiento y su incidencia en la calidad Integral universitaria", de la Universidad de Guayaquil en Ecuador, el mismo que busca optimizar los procesos académicos de la institución universitaria más grande del país que actualmente se encuentra intervenida por el Consejo de Educación Superior (CES), el ente regulador en el Ecuador. Así lo asevera Álvarez Pérez, (2014), mencionando que la calidad ofrecida en las instituciones educativas superiores (IES) debe estar en función de las competencias que exigen la vida laboral y social.

Según El Comercio (2016) se menciona que desde la primera intervención gubernamental que fue en el año 2013, la Universidad de Guayaquil ha experienciado una serie de cambios radicales en la gestión académica y administrativa, muchos de los indicadores de evaluación institucional han mejorado pasando de la categoría D a la B en septiembre del año 2016. Según el Reglamento para la Determinación de Resultados del Proceso de Evaluación, Acreditación y Categorización de las Universidades y Escuelas Politécnicas, regulado por la entidad existente en ese año, denominada Ceaaces, las Instituciones de Educación Superior (IES) ubicadas en la última categoría debían cumplir con el desarrollo de un plan de 
fortalecimiento institucional que les permita ascender y reubicarse, en la categoría más próxima, es decir la categoría C. La Universidad de Guayaquil pudo retomar su sitial en la categoría $B, y$ aunque a partir de ese momento, se dio fin a la primera intervención estatal, han pasado ya 3 años y la institución volvió a ser intervenida en octubre del 2018 y no ha podido alcanzar grandes mejoras en la calidad de la educación ni los resultados de excelencia que se pronosticaban en el 2016.

El área de titulación pertenece a una de las funciones sustanciales de la universidad, como lo es la Docencia, y para este criterio, los indicadores de gestión durante la primera intervención fueron bastante bajos, debido a que no se estaba aplicando correctamente el reglamento de régimen académico, que exige poseer unidades de titulación por cada carrera, y seleccionar modalidades para la misma. Consecuentemente, la tasa de eficiencia terminal, es decir la tasa de estudiantes egresados que lograron graduarse u obtener su título de tercer nivel, estaba en niveles realmente deplorables, con listados de estudiantes egresados de hasta 10 años atrás.

La Facultad de Ciencias Administrativas es una de las facultades más grandes de la Universidad de Guayaquil, con algo más del $20 \%$ de los 60 mil estudiantes que cursan sus carreras en las 18 facultades existentes. Esta unidad académica seleccionó dos principales modalidades de titulación, tales como el proyecto de emprendimiento y el trabajo de investigación. Un buen número de docentes descarga parte de sus horas académicas en el área de titulación, sin embargo, según datos preliminares, la selección de docentes es aleatoria, y no se consideran perfiles competentes para asignar horas de tutoría de titulación a los docentes adecuados. El objetivo de este estudio, es determinar el perfil idóneo del tutor de titulación de tercer nivel y así contribuir a la eficiencia y calidad de los procesos de elaboración de trabajos de titulación.

\section{REVISIÓN TEÓRICA}

El docente-tutor juega un rol pleno y estratégico en los contextos de la titulación de tercer nivel, ya que es quien realiza el seguimiento de la elaboración de los trabajos de titulación, así como parte de la evaluación de esto. De la misma manera, Ugarte y Naval, (2008) destacan que su papel es de alta importancia porque promueve diferentes estrategias de aprendizaje, de búsqueda bibliográfica, relativas a la investigación de campo y al análisis de resultados de investigación, adicionalmente es clave destacada en la motivación que recibe el estudiante para terminar su trabajo de titulación, que este mantiene un contacto continuo y 
permanente con sus tutoreados con la finalidad de orientar académicamente y que los estudiantes puedan superar los diversos obstáculos con los que se enfrentan.

El docente-tutor aporta un valor añadido a esta experiencia de la tutoría de titulación, ya que el conocimiento científico y profesional específico de este incrementa la eficacia de la tutoría y se visibiliza en las sesiones o entrevistas de asesoría. Esta actividad personalizada es la que promueve la mejora personal del estudiante y su orientación en el campo de la investigación, aportando a la mejora profesional e inclusive a su capacidad de encontrar el empleo adecuado luego de la culminación de su trabajo de titulación.

El proceso de tutoría arranca con la asignación tutor-estudiante, y para lo cual el tutor instruye al estudiante una serie de aspectos relativos a la incorporación de grado de tercer nivel y su salida universitaria frente al mundo real laboral, "lo que supone una orientación válida para superar la transición de la universidad al campo de trabajo" (Ocampo, 2012, p. 218).

Para Álvarez (2004) la función tutorial muchas veces se plantea erróneamente y ha servido para la mera descarga de horas de los docentes asignados, limitando su relevancia y recursividad, a la verificación de cumplimientos académicos, resolución de dudas en metodología de investigación, consultas diversas sobre formatos y estructuras de los trabajos de titulación, entre otras actividades más de carácter operativo y procesal. Los docentes tutores han olvidado la importancia de los aspectos personales del estudiante, de la motivación hacia la culminación de su carrera profesional y obtención del diploma acreditativo, además de que los trabajos de titulación podrían convertirse en el primer paso hacia la búsqueda de un empleo formal o del emprendimiento individual a través del diseño de propuestas realizables, de interés para las empresas que se desarrollan en el área de conocimiento del estudiante.

Además, la IES aplicó diferentes modalidades de tutorías, por ejemplo, se encuentra la tutoría académica, para lo cual Rillo (2015) considera que es un proceso, el mismo que acompaña al estudiante por parte de docentes competentes y profesionales. Desde esta perspectiva se puede concluir, que todo tipo de tutorías debe estar deacuerdo a los modelos educativos propuestos por el organismo regulador.

La valiosa figura del docente designado como tutor titulación, debe estar conformada por múltiples cualidades tanto personales como profesionales para que, en consecuencia, el acompañamiento de este al estudiante, sea óptimo y se consigan los resultados académicos necesarios para cumplir con los objetivos institucionales (Álvarez y González, 2008 citado por Romero, 2009). 
Las áreas de competencia del tutor podrían resumirse de la siguiente manera:

Figura 1. Áreas de competencia del Tutor.

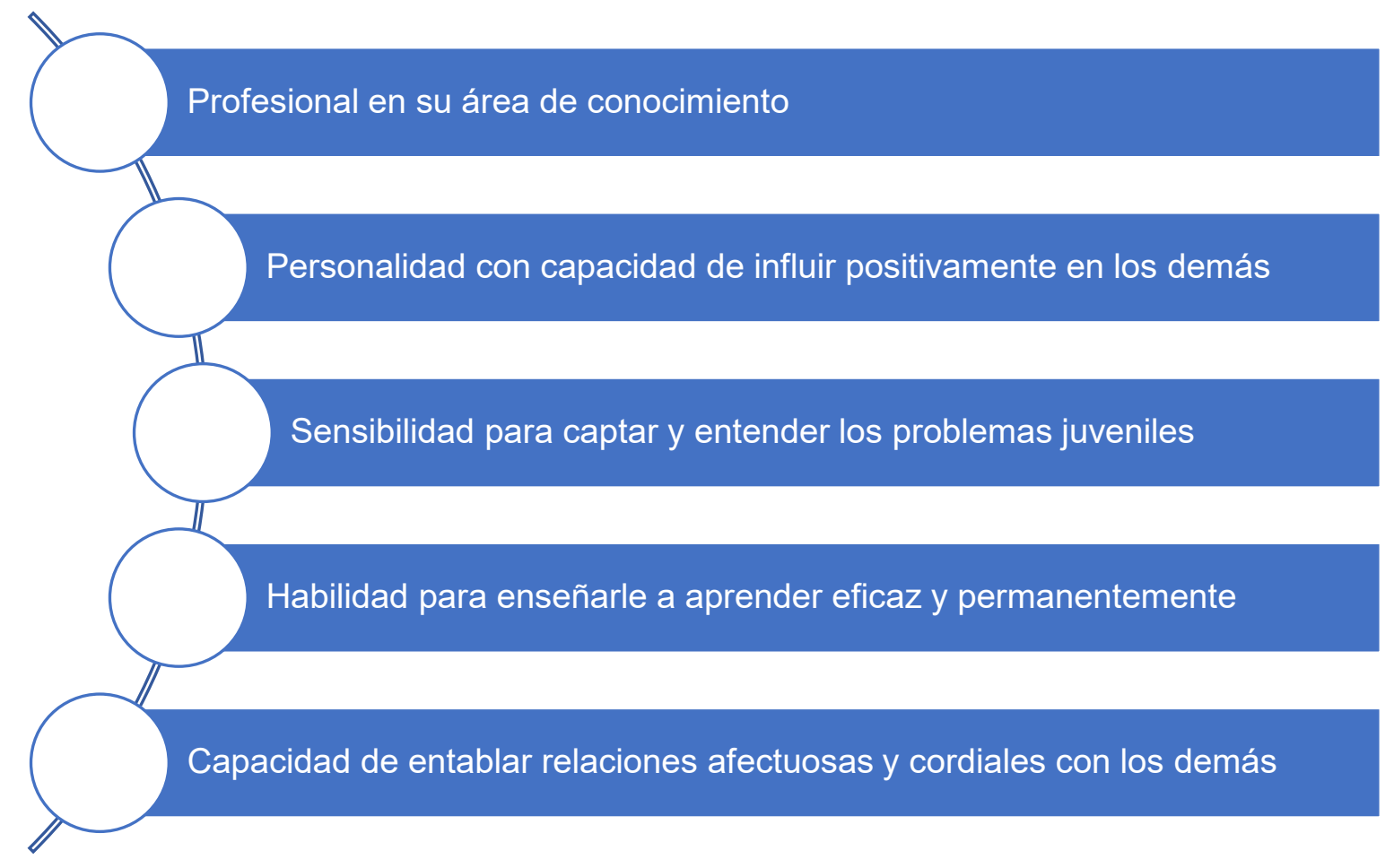

Fuente: Elaboración propia a partir de Ocampo (2012).

El perfil del tutor de titulación debe estar conformado por una serie de competencias, definidas como: "una combinación de destrezas, conocimientos y actitudes" (Morales, 2011, p. 18). Y así que estos sean los docentes idóneos para orientar a los estudiantes de forma rigurosa y sistemática en la culminación de sus trabajos de titulación de tercer nivel, y además evitar la deserción que en este punto conlleva mucho más riesgo de que la misma se vuelva indefinida debido a la realidad social del estudiantado de la Universidad de Guayaquil, que muchas veces se enfrasca en actividades laborales que no están relacionadas a sus estudios universitarios, pero que la presión de la situación económica existente puede convertirse en un factor de alta implicación.

En los contextos del área de titulación, el docente-tutor se convierte en un asesor, orientador, guía y evaluador de los procesos de investigación que lleva a cabo el estudiante. Por lo tanto, 
su dimensión tutorial prevalece sobre su dimensión docente. Se definen las siguientes acciones del docente-tutor.

Figura 2. Acciones del docente-tutor.

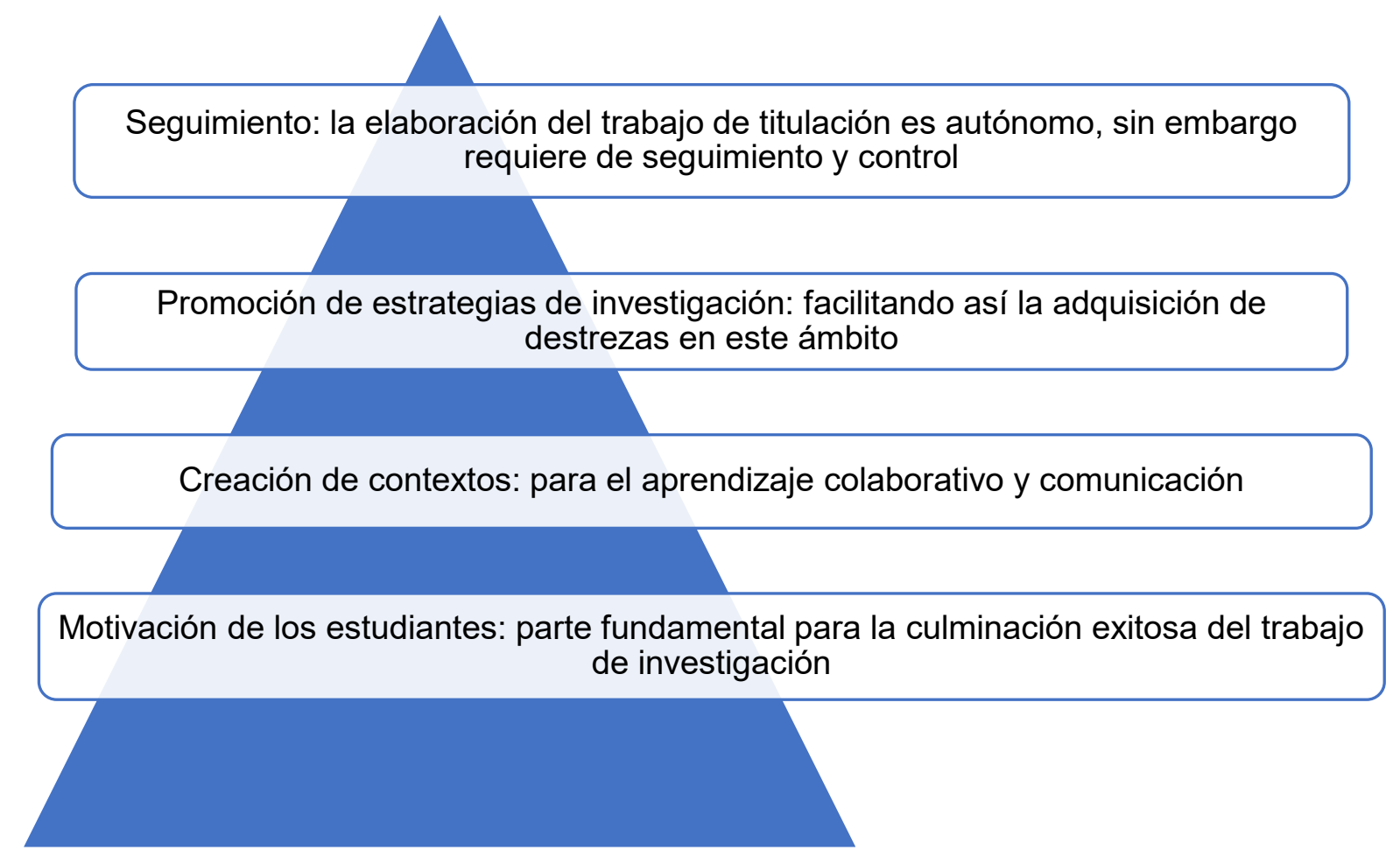

Fuente: elaboración propia a partir de Mir Repáraz y Sobrino (2003) citado por Ugarte y Naval (2008).

En definitiva, el papel del docente-tutor es vital en el seguimiento de los estudiantes que han optado por la modalidad de trabajo de titulación para obtener su diploma que lo acredite como profesional de la república ecuatoriana. El aprendizaje colaborativo es fundamental y la atención personalizada a sus estudiantes-tutorados es clave para el éxito de ambos. El docente-tutor deberá mantener una relación estrecha con los estudiantes asignados, los orienta en la elaboración de cada capítulo o sección de los trabajos de titulación, y tendrá conocimiento de las dificultades y obstáculos que el estudiante encuentra a lo largo del semestre (tiempo disponible para realizar el trabajo de titulación), además deberá conocer su grado de compromiso con las actividades propuestas.

Por lo tanto es necesario revelar la importancia de promover tutoría como función orientativa de estudiantes en su periodo de formación en la Universidad, así como en la elaboración de sus trabajos de titulación, pretendiendo que estos adquieran competencias de organización e 
investigación; sin embargo, si los tutores seleccionados por los directores de carrera no desarrollan una función de guías en estos aspectos es bastante complejo y dificultoso que se consigan los objetivos de la IES, así como el cumplimiento de indicadores de calidad en la gestión universitaria.

\section{MATERIALES Y MÉTODOS}

La presente investigación tiene como objetivo determinar el perfil del tutor de titulación del tercer nivel, él mismo que debe estar en función de sus competencias y habilidades. Para cumplir con este objetivo es necesario conocer la percepción de los docentes que fungen como tutores de titulación, es decir, aquellos que descargan horas de titulación dentro su franja horaria. Las evidencias empíricas demuestran que los docentes de titulación son los actores principales, no sólo por su experiencia, sino por el conocimiento de los procesos inmersos en el trabajo de titulación.

En concordancia, se puede inferir que los docentes de titulación aportarán con criterios o atributos que deben estar incluidos en el perfil profesional. Asimismo, los atributos serán clasificados en grupos homogéneos para su respectiva caracterización.

El enfoque de esta investigación es cualitativo, dado que la información recolectada se adecuará con la percepción del docente de titulación y por consiguiente el análisis respectivo. En este caso, se utilizará el método de expertos, el cual se basa en una comunicación estructurada y de predicción, es decir, a través de entrevistas a los docentes quienes conforman las plantillas en cada carrera, y así identificar las aptitudes que deben poseer.

Los datos se recogerán en la Universidad de Guayaquil, respectivamente en la ciudadela universitaria Salvador Allende, específicamente en los predios de la Facultad de Ciencias Administrativas (FCA).

La FCA posee ocho carreras, tales como: Contabilidad Pública Autorizada; Ingeniería en Marketing y negocios Internacionales, Ingeniería en Comercio Exterior, Gestión Empresarial, Ingeniería Comercial, Tributación y Finanzas, Ingeniería en sistemas de computación y Turismo. Es importante mencionar que desde el semestre pasado (ciclo II-2018-2019) en la FCA se han ofertado carreras de Licenciatura, acogiéndose al nuevo reglamento emitido por el Consejo de Educación Superior (CES). 
Las carreras mencionadas anteriormente están en un proceso de contingencia, es decir, cambio de malla de ingeniería a licenciatura, excepto la carrera de Turismo, por esta razón, solo se consideró a los docentes de las carreras que mantienen alumnos en el proceso de titulación.

Esta investigación, está dividida en dos partes, la primera se refiere a la utilización del focus group a los gestores de titulación de las siete carreras vigentes para otorgar títulos y a la coordinadora de titulación de la FCA, este instrumento permitirá identificar atributos que son aptos a cumplir por los tutores. El segundo punto, es la elaboración de una encuesta a los tutores, y así corroborar lo expuesto por los gestores y la coordinadora, y por consiguiente caracterizar las variables significativas.

La población será estudiada en su totalidad, de acuerdo a la investigación los docentes con horas de titulación asignadas en la FCA son 115.

\section{ANÁLISIS DE LOS RESULTADOS}

\section{1ra. etapa.}

Se realizó el focus group con los gestores de titulación y la coordinadora de este proceso, y se extrayeron 6 atributos que debe poseer un docente-tutor de titulación, con un 31\% debe poseer conocimiento, con un $27 \%$ debe ser competente, y con un $23 \%$ tiene que tener experiencia. Estos son los atributos con mayor importancia y los otros tres mencionados están por debajo del $10 \%$. Los resultados mostrados son la base para la elaboración de la encuesta a los docentes que descargan sus horas en el proceso de titulación. Se tomó solo los atributos con mayor frecuencia, en este caso el atributo conocimiento, competente y experiencia.

Tabla 1. Atributos que debe poseer un tutor de titulación.

\begin{tabular}{cc}
\hline Atributos & Frecuencia \\
\hline Conocimiento & $31 \%$ \\
Competente & $27 \%$ \\
Experiencia & $23 \%$ \\
Paciencia & $8 \%$ \\
Currículo & $8 \%$ \\
Responsabilidad & $4 \%$ \\
\hline
\end{tabular}

Fuente: Elaboración propia a partir de la información del focus group. 
2da. etapa.

En base a la información recolectada, se realizó una encuesta para caracterizar las primeras tres dimensiones obtenidas en la tabla 1 y se pudo concluir que: los docentes percibieron que el conocimiento es un atributo muy importante, ya que está en función de varias características tales como: el 30\% asevera que el docente debe tener estudios superiores de tercer nivel y cuarto nivel según la carrera, $32 \%$ opinan que la metodología de la investigación es la base de cualquier trabajo de titulación, $12 \%$ el conocimiento de las líneas de investigación, el 10\% el conocimiento de los instrumentos de análisis y con un $9 \%$ el conocimiento de las normas APA 6.

Tabla 2. Dimensión conocimiento.

\begin{tabular}{ccc}
\hline Dimensión & Frecuencia absoluta & \% \\
\hline Conocimiento de los estudios de la carrera & 46 & $37 \%$ \\
Metodología de la investigación & 40 & $32 \%$ \\
Líneas de investigación & 15 & $12 \%$ \\
Instrumentos de análisis de datos & 13 & $10 \%$ \\
Conocimiento en Normas APA & 11 & $9 \%$ \\
\hline Total & 125 & \\
\hline
\end{tabular}

Fuente: Elaboración propia.

En la tabla 3 se puede determinar que la percepción de los docentes hacia la dimensión competencia esté ligado a la producción científica por parte de los docentes. Con un $52 \%$ perciben que los docentes deben poseer artículos científicos en revistas regionales y de alto impacto. El $20 \%$ menciona que el docente es un guía o un asesor, y el $18 \%$ determina que el docente tiene experticia en el área temática.

Tabla 3. Dimensión Competente.

\begin{tabular}{ccc}
\hline Dimensión & Frecuencia absoluta & $\%$ \\
\hline Artículos científicos & 65 & $52 \%$ \\
Asesoría & 25 & $20 \%$ \\
Experticia en el área de temática & 22 & $18 \%$ \\
Participación en Ponencias & 13 & $10 \%$ \\
\hline Total & 125
\end{tabular}

Fuente: Elaboración propia. 
La tabla 4, se divide en dos criterios tales como: el número de trabajos de titulación y número de años como docente, en este caso los tutores percibieron: con un $68 \%$ que la experiencia esta medida con el número de trabajos y con un $32 \%$ esta medido por los años de docente.

Tabla 4. Dimensión Experiencia.

\begin{tabular}{ccc}
\hline Dimensión & $\begin{array}{c}\text { Frecuencia } \\
\text { absoluta }\end{array}$ & $\%$ \\
\hline Número de trabajos de titulación & 85 & $68 \%$ \\
Número de años como docente & 40 & $32 \%$ \\
\hline Total & 125 & \\
\hline
\end{tabular}

Fuente: Elaboración propia.

En resumen, se puede indicar que esta investigación permitió conocer tres dimensiones que deben estar inmersas en el perfil del tutor de titulación, este resultado permitió identificar criterios que corresponden a las dimensiones estudiadas según la percepción del docente que descarga horas de titulación en su jornada académica.

\section{CONCLUSIONES}

Los roles de docente-tutor y estudiante-tutorado han evolucionado ampliamente, y ambos poseen funciones activas en el proceso de elaboración de los trabajos de titulación, siendo la acción tutorial eje clave para la producción de los egresados de la Facultad de Ciencias Administrativas de la Universidad de Guayaquil.

El perfil del docente basado en la percepción de los docentes-tutores con horas de titulación en su jornada académica, responderá a mejorar el proceso de selección en el área de titulación y así favorecer al alumno en su última etapa estudiantil.

En este sentido, el perfil del docente se establece mediante los siguientes criterios: conocimiento, competencia y experiencia, tres ejes fundamentales que permitan transformar este proceso de titulación. Asimismo, existen criterios que se adecuan a cada uno de ellos. Entre los más representativos están el conocimiento de la metodología, estudios afines a la carrera a la que pertenecen, poseer producción científica, y tener años de experiencia como docente. Criterios que son percibidos adecuados los tutores de titulación. 


\section{REFERENCIAS BIBLIOGRÁFICAS}

Álvarez, P. (2014). La Función Tutorial del Profesorado Universitario: Una Nueva Competencia de la Labor Docente en el Contexto del EEES. Revista Portuguesa De Pedagogia, pp. 85-106. Acesso em de https://impactumjournals.uc.pt/rppedagogia/article/view/1907.

Álvarez, V. (2004). Las tutorías: otra forma de enseñar en la universidad. En La tutoría: elemento clave en el modelo europeo de Educación Superior. Universidad de Salamanca. Recuperado el 12 de julio, 2019 de http://campus.usal.es/ ofeees/PONENCIAS_TUTORIA/Victor\%20Alvarez\%20Rojo.p df

El Comercio (2016). La UG pasa de la categoría D a la B. Recuperado el 31 de julio, 2019 https://www.elcomercio.com/actualidad/universidad-guayaquil-categoriaeducacionsuperior-reneramirez.html

Gómez, M. E. (2012). La percepción de los estudiantes sobre el Programa de Tutoría Académica. Convergencia, 19(58), 209-233.

González, I. (2006). Dimensiones de evaluación de la calidad universitaria en el Espacio Europeo de Educación Superior. Revista electrónica de investigación psicoeducativa, 4(3), 445-468. http://unesdoc.unesco.org/images/0023/002312/231288e.pdf

LOES, (2010). Ley Orgánica de la Educación Superior. Suplemento del Registro Oficial No 298. Quito, Ecuador

Morales, J. C. (2011). Competencias y universidad, o un desajuste por mutua ignorancia. Bordón. Revista de pedagogía, 63(1), 15-31.

Ocampo, C.I. (2012). Tutoría Educativa en los diversos niveles y escenarios pedagógicos. En L.M. Sobrado; E. Fernández y M.L. Rodicio (Coords.), Orientación Educativa. Nuevas perspectivas (pp. 217-243). Madrid: Biblioteca Nueva.

Rillo, A. G. (2015). Solidaridad en la relación tutorial. Humanidades Médicas, 15(1), 4669. 
Romero, H. (2009). Sistemas Tutoriales en la Universidad. Jornadas Iberoamericanas de Tutoría y Orientación en Educación Superior. Universidad Maza (Mendoza).

Ugarte, C., y Naval, C. (2008). El profesor-tutor en una experiencia docente universitaria online-presencial. Teoría de la Educación. Educación y Cultura en la Sociedad de la Información, 9(1), 153-179.

Unesco (2014). Education Strategy 2014-2021.París. Recuperado el 25 de julio, 2019.

Veliz (2018). Calidad en la Educación Superior. Caso Ecuador. Atenas, Revista Científico-Pedagógica, Vol.1. No. 41-2018. Universidad de Matanzas, pp. 165-180. 